\title{
TOWARDS INTER-SUBJECT PROJECT-BASED LEARNING IN PROGRAMMING-RELATED COURSES AT COMPUTER SCIENCE STUDIES
}

\author{
Jordi Linares-Pellicer ${ }^{1}$, Jorge Orta-López ${ }^{1}$, José Salavert-Torres ${ }^{1}$, María José \\ Segura-Flor ${ }^{1}$, Joan Albert Silvestre-Cerdà ${ }^{1}$, Raquel Sanchis ${ }^{2}$ \\ ${ }^{1}$ Dpto. de Sistemas Informáticos y Computación, Escuela Politécnica Superior de Alcoy. \\ Universitat Politècnica de València (SPAIN) \\ ${ }^{2}$ Dpto. de Organización de Empresas, Escuela Politécnica Superior de Alcoy. Universitat \\ Politècnica de València (SPAIN)
}

\begin{abstract}
Curriculum design of University Degrees in Spain is mainly based on scheduling atomic, self-contained semester subjects during a 4-year period. This scheduling is driven by one major constraint: to ensure that previous subject pre-requisites are met for each course. Thus, basic subjects without college-level pre-requisites are typically scheduled in the first year, first semester, while the rest are properly planned in a sequential manner, complying the aforementioned condition. This is, basically, the only proof of inter-subject coordination in such degrees, taking place at the design stage. During the academic period, however, there is no vertical nor horizontal inter-subject coordination, not even among closely-related subjects.

In order to increase inter-subject coordination and to exploit its potential benefit for students, the projectbased learning (PBL) methodology shows on the scene. This approach organizes learning around student-driven projects aiming to solve real-life problems. This close applicability to the real world expedites intrinsic motivation of students, and consequently, their learning process tends to be deeper and more significant. Furthermore, PBL applied at the inter-subject level increases curriculum cohesion and makes students more engaged and compromised with its global objectives.

In this paper we describe the design of PBL models involving two pairs of programming-related subjects from the Computer Engineering Degree at the Universitat Politècnica de València. The first one associates the "Programming" with the "Data Structures and Algorithms" subject, while the second one engages the "Introduction to Video-Games Programming" with the "Digital Image Synthesis" one. It is an ambitious pilot programme that will require a high coordination effort among participating professors and student teams.
\end{abstract}

Keywords: project-based learning, computer science, programming.

\section{INTRODUCTION}

Curriculum design of University Degrees in Spain is mainly based on scheduling atomic, self-contained semester subjects during a 4-year period. This scheduling is driven by one major constraint: to ensure that previous subject pre-requisites are met for each course. Thus, basic subjects without college-level pre-requisites are typically scheduled in the first year, first semester, while the rest are properly planned in a sequential manner, complying the aforementioned condition. This is, basically, the only proof of inter-subject coordination in such degrees, taking place at the design stage. During the academic period, however, there is no vertical nor horizontal inter-subject coordination, not even among closely-related subjects.

In order to increase inter-subject coordination and to exploit its potential benefit for students, the projectbased learning (PBL) methodology shows on the scene. This approach organizes learning around student-driven projects aiming to solve real-life problems. This close applicability to the real world expedites intrinsic motivation of students, and consequently, their learning process tends to be deeper and more significant. Research has shown that students retain very little of what is taught in a traditional lecture format [1] and active methodologies, such as PBL, offers an appropriate alternative to traditional education by placing more emphasis on what the student learns than on what the professor teaches, and this results in greater understanding, motivation and participation of students in the learning process. Furthermore, PBL applied at the inter-subject level increases curriculum cohesion and makes students more engaged and compromised with its global objectives. 
The main objective of this paper is to define the design of PBL models involving two pairs of programming-related subjects from the Computer Engineering Degree at the Universitat Politècnica de València. The first one associates the "Programming" with the "Data Structures and Algorithms" subject, while the second one engages the "Introduction to Video-games Programming" with the "Digital Image Synthesis" one. It is an ambitious pilot programme that will require a high coordination effort among participating professors and students' teams.

The paper is organized as follows. Section 2 provides a general overview of the PBL foundations. Section 3 describes the two proposed PBL models. Finally, Section 4 provides some concluding remarks.

\section{PBL FOUNDATIONS}

PBL has become a topic of interest in order to face up to the current students' needs and the dynamic educational context. PBL is an instructional model based on having students confront real-world issues on problems that they find meaningful, determine how to address them, and then act in a fashion to create problems solutions [2].

PBL is considered as an effective instructional methodology that improves the levels of students engagement an achievements.

Projects must fulfil two fundamental criteria. The first aspect is that projects should make sense for students, that is, students must perceive it as something they personally want to do well because it is important and challenging. The second aspect is focused on the fact that the project should also have an educational purpose, it should be meaningful, according to the learning standards of the subjects involved in such a project. According to [3], the project on which students will work should include the following 8 elements:

1 Significant content. Professors should carefully plan the project in a way that focuses on the learning standards. From and education perspective, the project should reflect what is considered essential within the curriculum and from the students' viewpoint, they should find it meaningful, that is, real and close to their context and interests.

2 Need to know. An initial description of the context of the problem is required in order to make students question different aspects. The description of the problem should cause something emotional in the students, that is, something that activates student and appeals to their need to know.

3 Driving question. The question underlying the project should be provocative, open-ended, complex, and connected to the core of what students should learn. An appropriate driving question clearly captures the core of the project to make students perceive it as a challenge.

4 Voice and Choice. Once that the interest of the students has been captured, the professor could orientate and guide students in the definition of the following tasks to be carried out. However, there is a certain degree of students' autonomy, as they will be the responsible for proposing the project idea and/or solution. Students will share responsibilities among them and will manage deadlines appropriately.

5 21st Century Skills. Once the project is proposed, students work on it collaboratively. A feasible project should provide students with the possibility to practice and thus learn the skills demanded in these current times: critical thinking, effective communication, use of technologies, teamwork, among others.

6 In- Depth Inquiry. The investigation must follow a thread that leads students to question more issues, search for new resources and define their own conclusions and ideas about how to solve the problem of the project.

7 Revision and Reflection. The evaluation throughout the project by professors is very important, since they should supervise, check, and monitor the progress of the project. Furthermore, professor should promote constructive criticism among students.

8 Public audience. The results of the project should be presented to allow students to reflect on the work performed in the project once that it is finished. It is also important to encourage student to think about the future work lines and what they have learned.

Considering all these principles, section 3 is focused on the definition and design of the PBL models. 


\section{DEFINITION AND DESIGN OF THE PBL MODELS}

In this section we describe the design of two PBL models involving two pairs of programming-related subjects from the Computer Engineering Degree at the Universitat Politècnica de València. The first one, PBL1, involves the "Programming" (PRG) subject, scheduled at $1^{\text {st }}$ year, $2^{\text {nd }}$ semester of the study plan, and the "Data Structures and Algorithms" (DSA) subject, of $2^{\text {nd }}$ year, $2^{\text {nd }}$ semester. The second one, PBL2, engages the "Introduction to Video-games Programming" (IVP) subject with the "Digital Image Synthesis" (DIS) one, both scheduled for the $4^{\text {th }}$ year, $2^{\text {nd }}$ semester.

\subsection{Programming, Data Structures and Algorithms PBL Model}

The effective implementation of this PBL model will imply the execution of 9 activities. For each activity we specify the acronym of the subject, the type of classroom sessions, and the effective classroom hours devoted to them.

1 Learning the object-oriented paradigm [PRG, Theory, 12h]:

- During the classrooms we practise how to conceptually model objects into classes.

2 Analysis of linear data structures [PRG, Theory, 14h]:

- We study the basic linear data structures (stacks, queues and lists). Concretely we review its memory representations, method interface operations, common usage scenarios and related complexity analysis.

3 Analysis of a problem and application conceptual design [PRG, Lab, 4h]:

- The student is involved in the planning of a project. The student designs an application and the steps to create it. This application will address a problem using the object-oriented paradigm, so we also need to define the necessary objects and data structures (for example, an on-line catalogue of movies, series, books or videogames).

4 Implementation and adaptation of a linear data structure inside the project [PRG, Lab, 4h]:

- During several lab sessions theoretical knowledge is applied in order to implement a linear data structure. This data structure is integrated in the project's designed application, allowing to handle the collections of objects previously defined (movies, books...).

5 Analysis of advanced data structures [DSA, Theory, 6h]:

- More complex hierarchical data structures are studied. These complex data structures allow efficient data retrieval on big collections of objects (for example, prefix trees).

6 Analysis of advanced sorting algorithms [DSA, Theory, 2h]:

- Classroom lessons about advanced sorting algorithms. These algorithms allow sorting big collections of objects (for example, mergesort or quicksort).

7 Implementation and adaptation of an advanced data structure inside the project [DSA, Lab, 4h]:

- The student will implement more complex data structures in the project's application. Hierarchical data structures will allow to handle efficiently the collections modelled during the application conceptual design. The student must select a correct data structure depending on the problem to solve (for example, allowing more complex searches by author or title).

8 Implementation and adaptation of an advanced sorting algorithms inside the project [DSA, Lab, $2 h]$ :

- The student will further improve the software developed in his/her project with advanced sorting algorithms (for example, allowing sorting objects by antiquity, user score or relevancy).

9 Adaptation of the project to real use case scenarios [DSA, Lab, 2h]:

- The student will test the application against massive object collections obtained from real databases. The results of the tests will be included in the project's final report, analysing the efficiency and effectiveness of the student's application in different realistic scenarios.

Table 1 shows a chronogram with the scheduling of these nine activities. As this PBL model covers subjects scheduled in the $1^{\text {st }}$ and $2^{\text {nd }}$ years of the study plan, it has been set up as a 2-year PBL one. It will be interesting to study the effect of the significant time span that separate both subjects (half a year, approximately) on the effectiveness of this approach, in comparison with the PBL2 model that fully takes place in one semester. 
Table 1. Chronogram for the activities of the PBL1 model.

\begin{tabular}{|l|l|l|l|l|l|l|l|l|l|l|l|l|l|}
\hline \multicolumn{1}{c|}{} & \multicolumn{9}{c|}{2020} & \multicolumn{7}{c|}{2021} & \multicolumn{7}{c|}{} \\
\cline { 2 - 13 } \multicolumn{1}{c|}{} & 01 & 02 & 03 & 04 & 05 & 06 & $\ldots$ & 01 & 02 & 03 & 04 & 05 & 06 \\
\hline A1 & & & & & & & $\ldots$ & & & & & & \\
\hline A2 & & & & & & & $\ldots$ & & & & & & \\
\hline A3 & & & & & & & $\ldots$ & & & & & & \\
\hline A4 & & & & & & & $\ldots$ & & & & & & \\
\hline A5 & & & & & & & $\ldots$ & & & & & & \\
\hline A6 & & & & & & & $\ldots$ & & & & & & \\
\hline A7 & & & & & & $\ldots$ & & & & & & \\
\hline A8 & & & & & & & $\ldots$ & & & & & & \\
\hline A9 & & & & & & & $\ldots$ & & & & & & \\
\hline
\end{tabular}

\subsection{Video-Games Programming and Digital Image Synthesis}

Following the same criteria than in the previous PBL model, the different activities are defined. This PBL model also consists of 9 activities and for each one, we also present which subject/s is/are involved (its acronym), if the activity is related to theory classes (Theory) or to laboratory and practical classes (Lab) and the approximate length (in hours) devoted to each activity. The PBL2 model is defined as follows:

1 Learning the fundamentals of graphic and interactive application development environment [IVP+DIS, Theory + Lab, 4,5h]:

- This activity will be focused on showing the essentials of scene editing and creation tools, positioning and transformation of objects on stage, and fundamental types of objects in 2D and 3D environments to provide students with a solid basis for the project development.

2 Analysis of coding and storage of graphic elements [DIS, Theory + Lab, 4,5h]:

- In this activity, the student will study the different storage alternatives for vector and raster graphic elements, and data structures in polygonal modeling of surfaces.

3 Implementation of storage methods and visualization of polygonal models [DIS, Theory + Lab, 4,5h]:

- In this activity the student will apply the knowledge acquired in the procedural creation of geometric models and their visualization.

4 Analysis of geometric transformations and projection techniques [DIS, Theory + Lab, 4,5h]:

- This activity will provide theoretical foundations related to the main geometric transformations for the matrix implementation with high-level tools in interactive graphic environments.

5 Analysis of local and global lighting methods [DIS, Theory + Lab, 4,5h]:

- In this activity, the student will be able to identify the differences between local and global methods, light sources, shaders and post-processing.

6 Analysis of the differences in interactive applications and games of the kinematic and physical approaches [IVP, Theory + Lab, 4,5h]:

- In this activity, students will work from a theoretical as well as a practical perspective, kinematic techniques based on delta time, and analysis of characteristics and properties of a physical engine 
7 Implementation of visualization procedures through hierarchy or scene graph [IVP, Theory + Lab, 4,5h]:

- Students will have to develop scenes based on the use of parent / child hierarchies

8 Analysis of interaction techniques with elements in three-dimensional 3D scenes [IVP+DIS, Theory + Lab, 4,5h]:

- In this activity, the student will have to focus on the selection in three-dimensional space of objects by raycasting and their manipulation through different input elements.

9 Implementation of interactive prototypes or games with elements of procedural model generation [IVP+DIS, Theory + Lab, 4,5h]:

- In this final activity, the student will implement a complete prototype that combines the basic elements of procedural model generation and the integration of lighting and interaction techniques

Based on the previous activities, Table 2 shows the 9 activities planning. As both subjects are scheduled in the $4^{\text {th }}$ year of Computer Engineering, particularly in the second semester, the activities will be performed in parallel between both subjects, what implies a very high coordination of contents, sequences, times, and periods.

Table 2. Chronogram for the activities of the PBL2 model.

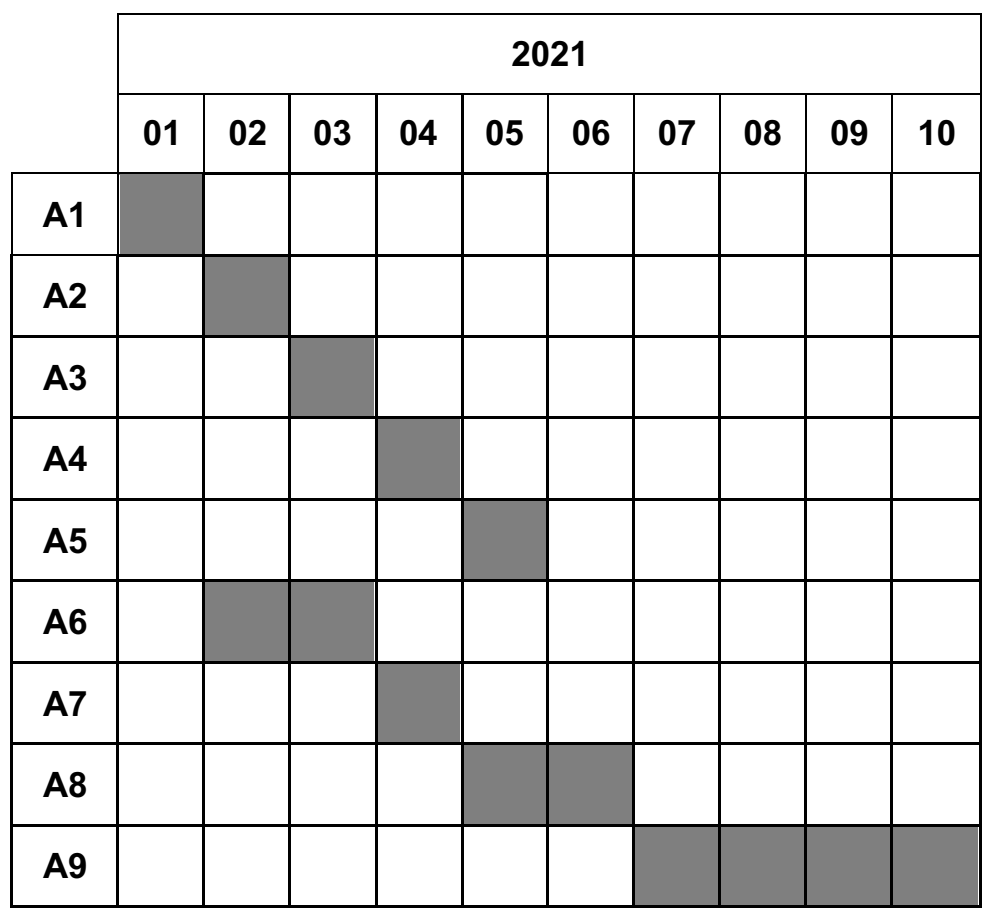

\section{CONCLUSIONS}

In this paper we have presented the design of two PBL models involving programming-related subjects from the Computer Engineering Degree at the Universitat Politècnica de València. The PBL1 model has already started with its first-year run, while the PBL2 model will start in the 2020-2021 academic course. We plan to report to the research community the results obtained in this innovative pilot programme, accompanied with a deep analysis on the strengthens and weaknesses found in the application of this approach.

One of the main challenges to which we face up is the current uncertainty due to the COVID19 breakout. At the moment that we send this contribution, Spanish Universities are finishing the second teaching semester, virtually. So far, the guidelines state that in September 2020, face-to-face classes will be resumed. However, these face-to-face classes will not be held under the same conditions than preCOVID19 as students' and lecturers' health should be guaranteed. All this situation causes great uncertainty, as it is unknown with certainty what type of teaching modality will be carried out next year. However, it is worth mentioning that the two PBL models presented can be implemented both in person 
and online modalities. The two PBL models are highly resilient and have a great adaptive capacity to fit these new and uncertain future conditions.

\section{ACKNOWLEDGEMENTS}

This article has been supported by the Vice-rectorate for Digital Resources and Documentation (Vicerrectorado de Recursos Digitales y Documentación) and Vice-Rectorate for Studies, Quality and Accreditation (Vicerrectorado de Estudios, Calidad y Acreditación) under the Call for Learning + Teaching (Convocatoria Aprendizaje + Docencia (A+D 2019)) and Project Code: 1678-A. The authors would like to acknowledge the support of the Institute of Educational Sciences (Instituto de Ciencias de la Educación) of Universitat Politècnica de València, the Evaluation and Monitoring Commission for Educational Innovation and Improvement Projects (Comisión de Evaluación y Seguimiento de Proyectos de Innovación y Mejora Educativa (CESPIME)) and Escuela Politécnica Superior de Alcoy.

\section{REFERENCES}

[1] B.J. Duch, S.E. Groh, D.E Allen. The Power of Problem Based Learning. Stylus 2001

[2] W. N. Bender. Project-based learning: Differentiating instruction for the 21st century. Corwin Press, 2012.

[3] J. Larmer and J.R. Mergendoller. PhD." 8 Essentials for Project-Based Learning.". 8 Essentials for Project-Based Learning, 1-4, 2012. 